\title{
SYMMETRY OF ELECTRONS AND HOLES IN LIGHTLY PHOTO-EXCITED
}

\section{InGaN LEDs}

\author{
T.A. Kennedy, * E.R. Glaser,* W.E. Carlos, ${ }^{*}$ P.P. Ruden** and Shuji Nakamura*** \\ *Naval Research Laboratory, Washington, DC 20375, kennedy@ bloch.nrl.navy.mil \\ **University of Minnesota, Department of Electrical and Computer Engineering, Minneapolis, \\ MN 55455 \\ ***Nichia Chemical Industries Ltd., Department of Research and Development, Tokushima, \\ JAPAN
}

Cite this article as: MRS Internet J. Nitride Semicond. Res. 4S1, G7.4(1999)

\begin{abstract}
The symmetry of the recombining electrons and holes in lightly photo-excited InGaN LEDs revealed through ODMR is related to the physical structure, band structure and defects present. Calculations of the electron-g within the $\boldsymbol{k} \bullet \boldsymbol{p}$ formalism give the average shift from the freeelectron value for $\mathrm{GaN}$ but are not fully reconciled with the anisotropy. This theory is also extended to InGaN alloys for both pseudomorphic and relaxed layers. The average shift is close to the experimental values for the green LED. The strongly reduced hole anisotropies seen experimentally are explained by a recently published theory for acceptors in GaN.
\end{abstract}

\section{INTRODUCTION}

Advances in nitride materials have led to the development of state-of-the-art LEDs in the visible range and lasers in the near uv [1]. While the success of these nitride devices has been remarkable, there is a need for an improved understanding of the recombination process. Interest at the moment is focused on the influence of the spontaneous polarization, the piezoelectric effect arising mismatch-induced strain and the potential fluctuations associated with the tendency in InGaN alloys toward phase separation. Numerous studies of these effects have been published. Among the optical characterizations are studies of photoluminescence, photoluminescence excitation and photoluminescence decay [1-5]. These studies reveal the effects of charge separation of the electron and hole in the growth direction and localization of the carriers or excitons by potential fluctuations in the growth plane.

Optically detected magnetic resonance (ODMR) has recently been observed in green and extrablue InGaN single-quantum well diode structures [6]. The distinct electron and hole resonances denote significant charge separation in the lightly photo-excited structures. Analysis of the frequency dependence of the ODMR for magnetic-dipole microwave transitions gives long lifetimes [7]. The ODMR technique is particularly valuable since the g-factors directly reflect the symmetry of the recombining electron and hole. This symmetry reveals the detailed structure determining the electron- and hole-states.

This paper explores the link between the symmetry as revealed by ODMR and the vertical and lateral structure of the InGaN LED. Theories for the g-factor of electrons and localized holes are presented, extended and compared with experiment. For the electron, the present extensions of the theory are still inadequate to achieving a good understanding of the data. For the hole, new spectral data is presented and discussed using a recent theory for acceptors in GaN [8]. The analysis shows that the hole states are affected by strong in-plane strain. 


\section{SYMMETRY OF THE ELECTRON}

The g-factor for a conduction electron in a direct-gap semiconductor can be calculated using $\boldsymbol{k} \bullet \boldsymbol{p}$ theory [9]. The g-values for $\mathrm{GaN}$ were calculated within a cubic approximation [10]. It is necessary to include both the valence bands and the upper conduction bands in order to achieve agreement with experiment. The experiments in InGaN LEDs show changes in the average gfactor and in the anisotropy of the g-factor[6]. Hence it is necessary to extend the calculations to include the wurtzitic crystal fields, the changes in band structure in moving from $\mathrm{GaN}$ to the InGaN alloy, and effects due to the quantum well and differing barrier layers. The following paragraphs address these issues.

First, the anisotropic g-tensor for electrons in wurtzitic $\mathrm{GaN}$ is calculated. Two sets of band parameters were chosen [11]. The first is a "best" set from a mix of theory and experiment within a quasi-cubic approximation. The second is a full set of calculated values. The energy differences, crystal-field and spin-orbit splittings and interband matrix elements are shown in Fig. 1 and collected in Table 1. For coupling between the lowest conduction band and the uppermost valence band, the expressions for the g-factors are

$$
\begin{aligned}
& \frac{g_{\|}}{g_{0}}-1=\frac{-P^{2}}{2}\left\{\frac{1}{E_{0}-E_{A}}-\frac{\gamma_{1}^{2}}{E_{0}-E_{B}}-\frac{\left(1-\gamma_{1}^{2}\right)}{E_{0}-E_{C}}\right\} \quad \text { and } \\
& \frac{g_{\perp}}{g_{0}}-1=\frac{-P^{2}}{2}\left\{\frac{1}{E_{0}-E_{B}}-\frac{1}{E_{0}-E_{C}}\right\} \sqrt{2} \cdot \gamma_{1} \sqrt{1-\gamma_{1}^{2}}
\end{aligned}
$$

where

$$
\begin{gathered}
\gamma_{1}=\frac{\gamma}{\sqrt{\gamma^{2}-1}}, \quad \gamma=\frac{\Delta_{1}-\Delta_{2}+\sqrt{\left(\Delta_{1}-\Delta_{2}\right)^{2}+8 \Delta_{3}^{2}}}{2 \sqrt{2} \Delta_{3}} \quad \text { and } \\
E_{A}=\Delta_{1}+\Delta_{2} \quad E_{B}=\frac{1}{2}\left\{\left(\Delta_{1}-\Delta_{2}\right)+\sqrt{\left(\Delta_{1}-\Delta_{2}\right)^{2}+8 \Delta_{3}^{2}}\right\} \\
E_{C}=\frac{1}{2}\left\{\left(\Delta_{1}-\Delta_{2}\right)-\sqrt{\left(\Delta_{1}-\Delta_{2}\right)^{2}+8 \Delta_{3}^{2}}\right\}
\end{gathered}
$$

Similarly, the contributions from the upper conduction bands were calculated using the same equations with the upper conduction band parameters replacing the valence band contributions. The contributions from both bands add and are of the same magnitude. The resulting total gfactors are given in Table 2 for both the "best" parameters and the calculated parameters. The 


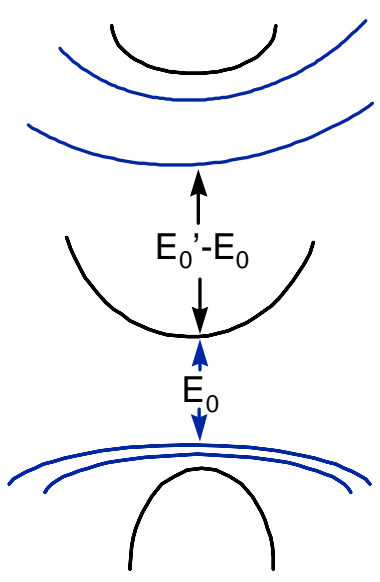

Figure 1. Band structure for wurtzitic nitrides.

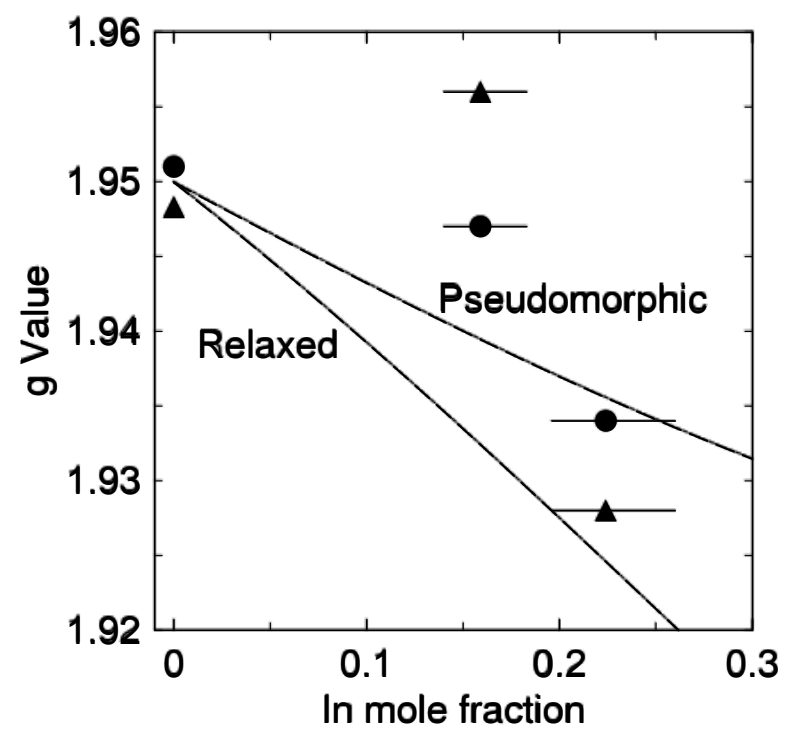

average shift from the free-electron g-factor is predicted well. The anisotropy is of the right order but with differing signs: disagreeing with experiment for the "best" parameters but with the right sense for the calculated parameters. Thus the anisotropy in wurtzitic GaN depends in a delicate way on the band parameters.

Table 1. Band parameters used in the $\boldsymbol{k} \bullet \boldsymbol{p}$ calculations.

\begin{tabular}{lllllllllll}
\hline & $\mathrm{E}_{0}$ & $\Delta_{1}$ & $\Delta_{2}$ & $\Delta_{3}$ & $\mathrm{P}^{2}$ & $\mathrm{E}_{0}{ }^{\prime}$ & $\Delta_{1}{ }^{\prime}$ & $\Delta_{2}{ }^{\prime}$ & $\Delta_{3}{ }^{\prime}$ & $\mathrm{P}^{\prime 2}$ \\
\hline Best & $3.5 \mathrm{eV}$ & 10 & 6.3 & $(6.3$ & 22.5 & 9.015 & -0.406 & 0.112 & $(0.112$ & $9 \mathrm{eV}$ \\
$\mathrm{GaN}$ & & $\mathrm{meV}$ & $\mathrm{meV}$ & $\mathrm{meV})$ & $\mathrm{eV}$ & $\mathrm{eV}$ & $\mathrm{eV}$ & $\mathrm{eV}$ & $\mathrm{eV})$ & \\
$\begin{array}{l}\text { Calcul } \\
\text { ated }\end{array}$ & 36 & $\begin{array}{l}5.0 \\
\mathrm{meV}\end{array}$ & $\begin{array}{l}5.9 \\
\mathrm{meV}\end{array}$ & & & & & & \\
$\mathrm{GaN}$ & & & & $\mathrm{meV}$ & & & & & & \\
$\mathrm{InN}$ & $\begin{array}{l}1.89 \\
\mathrm{eV}\end{array}$ & $\begin{array}{l}43 \\
\mathrm{meV}\end{array}$ & $\begin{array}{l}0.3 \\
\mathrm{meV}\end{array}$ & $\begin{array}{l}0.3 \\
\mathrm{meV}\end{array}$ & $\begin{array}{l}22.5 \\
\mathrm{eV}\end{array}$ & $\begin{array}{l}7.91 \\
\mathrm{eV}\end{array}$ & & $\begin{array}{l}0.284 \\
\mathrm{meV}\end{array}$ & $\begin{array}{l}0.284 \\
\mathrm{meV}\end{array}$ & $9 \mathrm{eV}$ \\
\hline
\end{tabular}

Table 2. Electron g-values in wurtzitic GaN.

\begin{tabular}{ccc}
\hline & $\mathrm{g}_{\|}$ & $\mathrm{g}_{\perp}$ \\
\hline Experiment & 1.9510 & 1.9483 \\
Best & 1.952 & 1.953 \\
Calculated & 1.955 & 1.954 \\
\hline
\end{tabular}

In previous work the g-values have been calculated for In-mole fractions up to 0.4 [6,7]. The calculations were done for unstrained alloys assumed to be cubic. Here these calculations are improved by incorporating the recent results for the energy-gap dependence on alloy composition [12]. The new relationship allows a comparison of the g-factors with calculations for both pseudomorphic and fully relaxed InGaN. 
The new calculations were done by combining the gap equation with a $\boldsymbol{k} \bullet \boldsymbol{p}$ calculation within a quasi-cubic approximation. The equation for the energy gap is

$$
E_{G}=3.42(1-x)+1.89 x-b x(1-x) \text {, }
$$

where the bowing parameter, $\mathrm{b}$, is $2.6 \mathrm{eV}$ for a pseudomorphic layer and $3.8 \mathrm{eV}$ for a fully relaxed layer. Since the original estimates for $x$ for the green and extra-blue diodes were made with a bowing parameter of $1 \mathrm{eV}$, these need to be revised. The green changes from 0.43 to 0.22 and the extra-blue from 0.3 to 0.16 . The band parameters for $\mathrm{InN}$ are shown in Table 1. The interband matrix elements are taken from $\mathrm{GaN}$. $\mathrm{E}_{0}$ ' and $\Delta_{2}$ ' are scaled from $\mathrm{GaN}$ using the ratio of the values of atomic transitions. $\Delta_{1}$ and $\Delta_{2}$ for InN are taken from first-principles calculations [13]. For the pseudomorphic layers, strain introduces a $\Delta_{1}$ by a deformation potential taken also from the first-principles calculations. $\Delta_{1}, \Delta_{2}, \Delta_{2}$ ' and $E_{0}$ ' are then scaled to different In mole fractions by a virtual crystal approach. The g-shifts versus mole-fraction obtained in this way needed to be scaled by $15 \%$ to match at GaN.

The calculated g-factors versus mole fraction for both relaxed and pseudomorphic layers are plotted along with the data in Fig. 2. The experimental values for the green diode, expected to be relaxed, fall close to the calculated values. However, the g-values for the extra-blue diode lie substantially above the calculated lines. From the reversal in anisotropy for this structure and its lower mole-fraction, the extra-blue diode is expected to be pseudomorphic. Thus while the calculation approximates the g-factors for the green diode, other interactions must be important for the extra-blue diode.

The next step in this analysis is to consider the effect of the quantum well potential. Theories have been developed for other heterostructure systems [14]. The strain, confinement and piezoelectric effect present in the InGaN LED structure certainly provide enough interactions to produce the reversal in symmetry observed experimentally. Hence, it does seem likely that a clear understanding of the symmetry of the electronic state can be deduced from the experimental data.

\section{SYMMETRY OF THE HOLE}

The g-factors for an acceptor in a wide-bandgap semiconductor have been calculated [8] for an acceptor including both Coulomb and central-cell potentials. Formulation of the problem in kspace avoids a divergence at the origin. The calculation is first done in a spherical approximation with only the Coulomb and central-cell potentials. The Coulomb interaction gives a binding energy of $200 \mathrm{meV}$. The crystal field is introduced as a strain in the [111] direction which corresponds to a crystal-field splitting of $37 \mathrm{meV}$. Spin-orbit interaction is then added to produce a splitting of $12 \mathrm{meV}$. This reproduces the conditions in GaN with perfect crystal structure and leads to highly anisotropic g-values. Since previous ODMR studies of acceptors in GaN revealed g-tensors which are only weakly anisotropic [15,16], the authors then consider an additional strain which lowers the symmetry at the acceptor from axial to rhombic and produces a reduction in the g-anisotropy. The authors attribute the reduction in symmetry to the Jahn-Teller effect but the symmetry lowering could arise from other sources.

The results of this theory can be summarized by considering the g-factors as a function of the symmetry-lowering strain energy (See Fig. 3). For an acceptor in purely axial symmetry $\mathrm{g}_{\|}$is 3.3 and $g_{\perp}$ is 0 . In-plane strain becomes important as its associated energy becomes comparable to the spin-orbit splitting of $12 \mathrm{meV}$. For large in-plane strain, the g-tensor becomes less anisotropic and reverts to the free-electron value of 2.00 . 
When all the luminescence is collected from the green LED, the g-tensor for the hole is $\mathrm{g}_{\|}=2.08$ (1) and $g_{\perp}=1.996$ (3)[6]. The anisotropy is strongly reduced from that expected for holes in purely axial symmetry. Using filters, a spectral study has been performed which reveals that the $\mathrm{g}_{\|}$changes for emission at different energies (See Fig. 4). In the study, long-pass filters were used to detect all the emission across the green band and the emission from the lower-energy half of the band. A subtraction reveals the part characteristic of the upper energy-range with $\mathrm{g}_{\|}=$ 2.20 and the one filter gives the lower range with $\mathrm{g}_{\|}=2.09$. There is a direct correlation between decreasing energy and decreasing g-value. This may arise in part from a changing hole g-factor with changing In-concentration. However, the character of the hole may also be changing. Since a deepening in energy is expected with increasing in-plane strain, the reduction in anisotropy with energy may also arise from increased in-plane strain.

In summary, the g-tensor for holes in lightly photo-excited InGaN reveals states which differ from those of the band edge and those for bound holes in purely axial symmetry. The binding could arise from either an acceptor or a fluctuation in composition of the alloy. The theory which describes the changes in g-tensor for different values of in-plane strain provides a solid framework to interpret the data. Spectral studies of the g-factor show that the anisotropy decreases with decreasing emission-energy. Compositional change and greater non-axial strain produce the reduction in anisotropy. These results are consistent with the behavior of acceptors in other semiconductors [17].
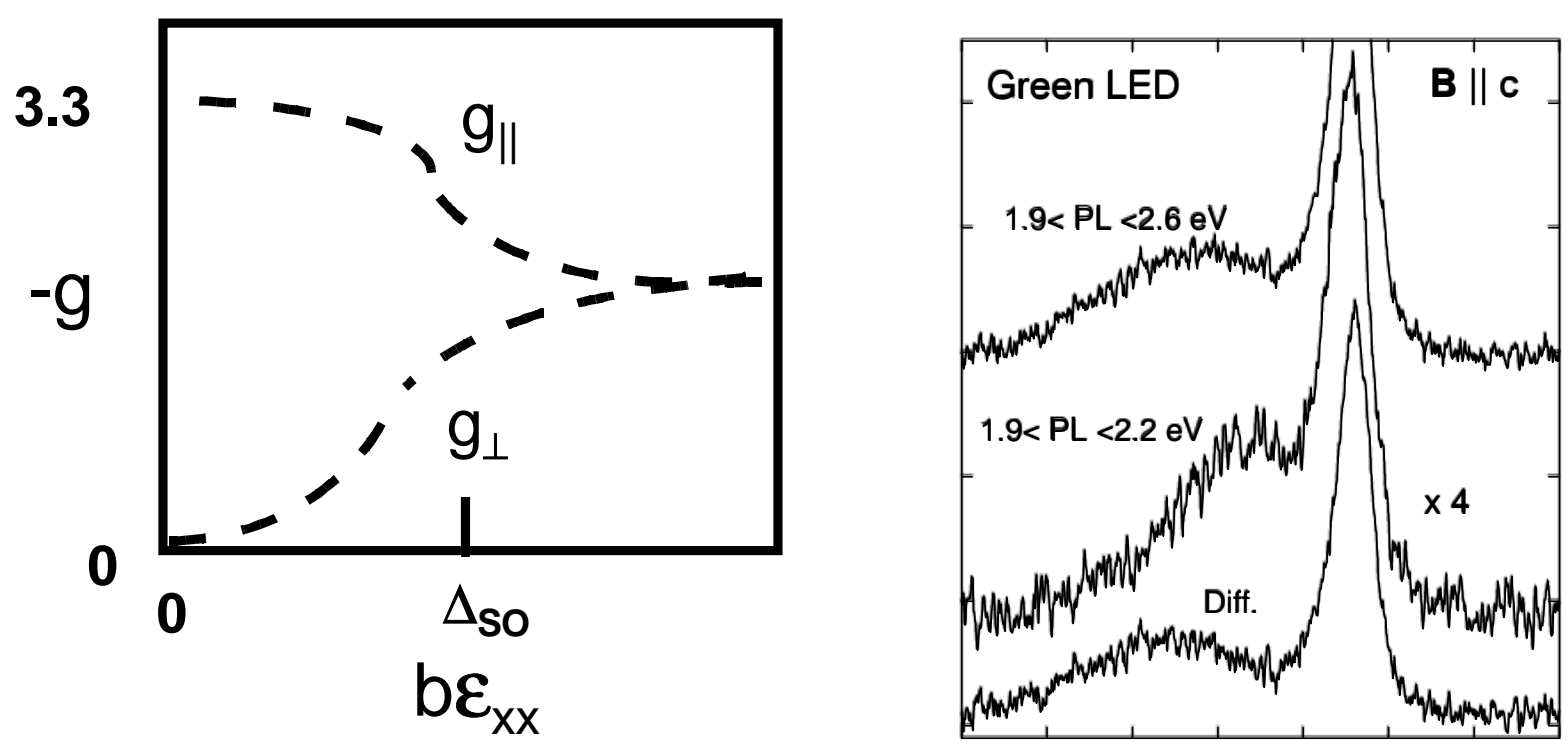

Figure 3. Hole g-factors in $\mathrm{GaN}$ versus the energy associated with an in-plane strain. The measure of the energy is the spin-orbit splitting.
Figure 4. Spectral decomposition of the ODMR for the Green LED. The two experimental curves are subtracted to give the difference spectrum. 


\section{SUMMARY}

The symmetry of the g-factors of recombining electrons and holes in InGaN is related to the band structure and local perturbations of that structure. For GaN, the average g-factor for electrons can be deduced from known band parameters but the anisotropy is difficult to calculate. Improved calculations are made for both pseudomorphic and relaxed InGaN. Again the anisotropy is beyond the current calculations. The hole g-anisotropy is strongly reduced from that for the band edge. A recent theory for acceptors in $\mathrm{GaN}$ demonstrates the experimental features and reconciles them with strong in-plane strain.

\section{ACKNOWLEDGEMENTS}

The work at NRL was supported by ONR. We thank I. Merkulov for helpful conversations. PPR thanks W.R.L. Lambrecht for unpublished GaN band structure data.

\section{REFERENCES}

1. Nakamura and Fasol, The Blue Laser Diode (Springer, Berlin, 1997).

2. K.P. O'Donnell, T. Breitkopf, Hl.Kalt, W. Van Der Stricht, I. Moerman, P. Demeester and P.G. Middleton, Appl. Phys. Lett. 70, 1843 (1997).

3. S.F. Chichibu, A.C. Abare, M.S. Minsky, S. Keller, S.B. Fleischer, J.E. Bowers, E. Hu, U.K. Mishra, L.A. Coldren, S.P. DenBaars and T. Sota, Appl. Phys. Lett. 73, 2006 (1998).

4. Yong-Hoon Cho, G.H. Gainer, A.J. Fischer, J.J. Song, S. Keller, U.K. Mishra and S.P. DenBaars, Appl. Phys. Lett. 73, 1370 (1998).

5. T.J. Schmidt, Yong-Hoon Cho, G.H. Gainer, J.J. Song, S. Keller, U.K. Mishra and S.P. DenBaars, Appl. Phys. Lett. 73, 1892 (1998).

6. E.R. Glaser, T.A. Kennedy, W.E. Carlos, P.P. Ruden and S. Nakamura, Appl. Phys. Lett. 73, 3123 (1998).

7. W.E. Carlos, E.R. Glaser, T.A. Kennedy and Shuji Nakamura, J. Electron Mater., to be published.

8. A.V. Malyshev, I.A. Merkulov and A.V. Rodina, Phys. Solid State 40, 917 (1998).

9. C. Hermann and C. Weisbuch in Optical Orientation, F. Meier and B.P. Zakharchenya, editors (North Holland, Amsterdam, 1984), p. 463.

10. W.E. Carlos, J.A. Freitas, Jr., M. Asif Khan, D.T. Olson and J.N. Kuznia, Phys. Rev. B 48, 17,878 (1993).

11. Kwiseon Kim,Walter R.L. Lambrecht, Benjamin Segall and Mark van Schilfgaarde, Phys. Rev. B 56, 7363 (1997).

12. C. Wetzel, T. Takeuchi, S. Yamaguchi, H. Katoh, H. Amano and I. Akasaki, Appl. Phys. Lett. 73, 1994 (1998).

13. Su-Huai Wei and Alex Zunger, Appl. Phys. Lett. 69, 2719 (1996).

14. E.L. Ivchenko and A.A. Kisilev, Sov. Phys. Semicond. 26, 827 (1992).

15. M. Kunzer, J. Baur, U. Kaufmann, J. Schneider, H. Amano and I. Akasaki, Solid-State Electron. 41, 189 (1997).

16. E.R. Glaser, T.A. Kennedy, K. Doverspike, L.B. Rowland, D.K. Gaskill, J.A. Freitas, Jr., M. Asif Khan, D. T. Olson, J.N. Kuznia and D.K. Wickenden, Phys. Rev. B 51, 13,326 (1995).

17. T.N. Morgan, Proceedings of the Tenth International Conference on the Physics of Semiconductors (U.S. Atomic Energy Commission, 1970), p. 266. 